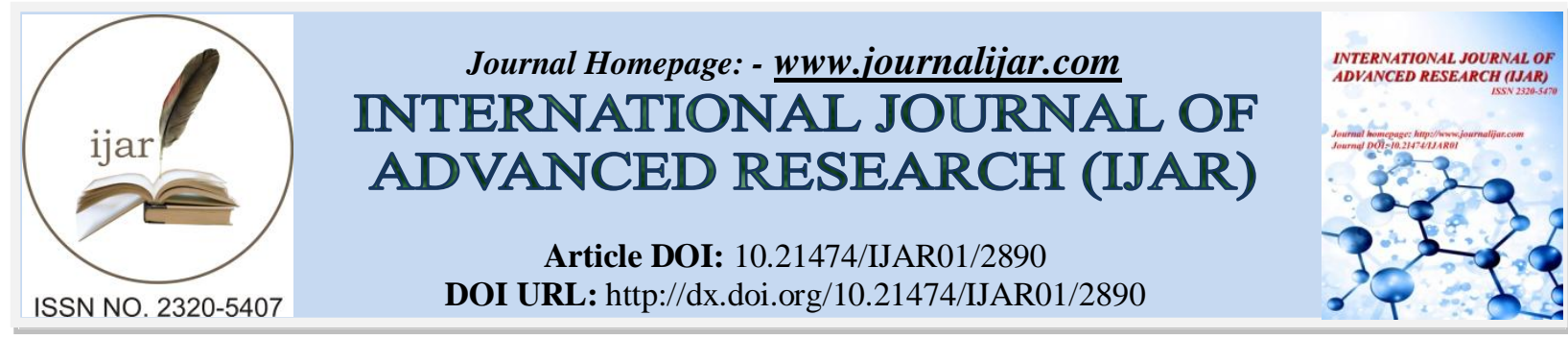

RESEARCH ARTICLE

\title{
VARIABILITY OF MESENCHYMAL VEGFR 1, 2, AND 3 PROTEIN EXPRESSION OFHUMANS AND MURINE SPECIES - A COMPARATIVE ANALYSIS.
}

\author{
Ahmed Alrabai ${ }^{1}$, Ahmed AL-Hakami ${ }^{1,2}$ and Harish C Chandramoorthy ${ }^{1,2}$. \\ 1. Center for Stem Cell Research, College of Medicine, King Khalid University, Abha, Saudi Arabia \\ 2. Department of Microbiology and Parasitology, College of Medicine, King Khalid University, Abha, Saudi \\ Arabia
}

\section{Manuscript Info}

\section{Manuscript History}

Received: 22 November 2016

Final Accepted: 23 December 2016

Published: January 2017

Key words:-

Vascular Endothelial Growth Factors,

VEGFR 1, VEGFR 2, VEGFR 3, Protein

Phylogenetic Analysis, Amino Acid

Sequences.

\begin{abstract}
The Vascular Endothelial Growth Factor 1,2,3 (VEGFR 1,2,3) protein sequences from various species like Mus musculus (Mouse), Rattus norvegicus (Rat) and Homo sapiens (Human) were retrieved from SWISS PROT database and compared for their homology and variability.DNAMAN Software was used to create multiple sequence alignment and in assessment of the homology and variability. The results of the phylogenetic tree assessment for VEGFR1, 2, 3 depicts that the sequences from Mus musculus (Mouse) and Rattus norvegicus (Rat) showed high homology when compared with the sequences of the Homo sapiens (Human). The intra sequence homology within the humanVEGFR1,2,3 protein predicted and analyzed for secondary structure show a characteristic variability in the amino acid positions on the random coils, alpha confirmationsand extended strands without significant variation in the length.
\end{abstract}

Copy Right, IJAR, 2016,. All rights reserved.

\section{Introduction:-}

Stem cells are totipotent primordial cells capable to differentiate into any known adult cell types with its typical physiological functions (Louveau I et al., 2016). The first stem cells originate within the developing embryo (blastocyst) and have two important characteristics that distinguish them from other types of cells. First, they are unspecialized cells that renew themselves for long periods through cell division. The second is that, under physiologic or experimental conditions, they can be induced to become cells with special functions such as the beating cells of the heart muscle or the insulin-producing cells of the pancreas (Adel Alhadlaq and Jeremy J. Mao, 2004).Stem Cells reside as resident population at various organs and can be isolated from variety of tissues like adipose tissue, bone marrow, embryos, olfactory ensheathing cells, peripheral blood, skin and umbilical cord blood.Further stem cells or progenitor cells can be mobilized form bone marrow upon signaling from the infracted or damages tissues.

There are different types of stem cells like embryonic stem cells, endothelial stem cells, mesenchymal or stromal stem cells, hematopoietic progenitors, cardiac, cancer and neuronal stem cells, induced pluripotent stem cells etc (Choudhery MS et al., 2015). All these have been isolated, banked or used for research with few successful clinical applications. However identification of these cell with their characteristic phenotypic or genotypic markers are more difficult and challenging today. There are surface markers common for these cells with few

Corresponding Author:- Harish C Chandramoorthy.

Address:- Center for Stem Cell Research, College of Medicine, King Khalid University, Abha, Saudi Arabia and Department of Microbiology and Parasitology, College of Medicine, King Khalid University, 
exceptions like common leucocyte markers like CD45 which is mostly observed only in the cells of the hematopoietic origin (Kobolak et al., 2016). Further many progenitor stem cells usually shares surface markers making it difficult to isolate or characterize as functional lineages, though these markers are very particular for some discrete functional adult differentiated cells. Therefore the identification and characterization of the stem cells with the available markers or cluster of differentiation (CD) on the surface of these naïve cells becomes more difficult and generalized rather than specific. Therefore it becomes necessary to eliminate such older cells based on the other markers characteristic to the anatomical site of the cells or type of the cells. The best example of these cases isVascular Endothelial Growth Factor(VEGFR) group of the markers which are very specific to endothelial progenitors or endothelial cells (Igarashi $Y$ et al., 2016). The presence of VEGFR has been well characterized in the mesenchymal stem cells too (Totsugawa T et al., 2002), though MSCs functions are much varied.The VEGFRfamily consists of three members, Vascular Endothelial Growth Factor Receptor -1 (Flt-1), Vascular Endothelial Growth Factor Receptor -2 (KDR/Flk-1) and Vascular Endothelial Growth Factor Receptor -3 (Flt-4), all of which belong to the receptor type tyrosine kinase superfamily (Matthias Clauss, et al., 1996, Kasper $\mathrm{G}$ et al., 2007,). In the current investigation we intended to examine the variability of VEGFR1,2 and 3 among the experimental animals and human origin. We tend to investigate the degree of identity among human and animals and within animal groups. The VEGFR 1,2 and 3 amino acid sequences from human origin and from the murine origin (Mouse and Rat) were assessed for the homogeneity and difference in their protein confirmation. This will the give the information on the degree of the cross reactivity of the VEGFR antibodies against VEGFR 1,2,3 and species cross reactivity with murine origin.

\section{Materials and Methods:-}

Retrieval of sequences:-

The amino acid sequences of the surface marker Vascular Endothelial Growth Factor Receptor (VEGFR) was retrieved using NCBI and SWISS-PROT. Sequences of all three receptors, namely, VEGFR1, VEGFR2 and VEGFR3 from different species such as Homo sapiens (Human), Mus musculus (Mouse) and Rattus norvegicus (Rat) were used for this study.

SWISS-PROT (http://www.expasy.org/sprot/)(Amos Bairoch and Rolf Apweiler, 2000) Swiss-Prot is a curated biological database of protein sequences from different species created in 1986 by Amos Bairoch during his doctoral work and developed by the Swiss Institute of Bioinformatics and the European Bioinformatics Institute. It strives to provide a high level of annotation (such as the description of the function of a protein, its domains structure, posttranslational modifications, variants, etc.), a minimal level of redundancy and high level of integration with other databases.Now funded by the NIH, Swiss-Prot and its automatically curated supplement TrEMBL have joined with the PIR to produce the UniProt Knowledgebase, the world's most comprehensive catalogue of information on proteins. It is a central repository of protein sequence and function created by joining the information contained in Swiss-Prot, TrEMBL, and PIR.

NCBI-ENTREZ (http://www.ncbi.nlm.nih.gov/) (Sayers EW and Karsch-Mizrachi I, 2016)

Established in 1988 as a national resource for molecular biology information, NCBI creates public databases, conducts research in computational biology, develops software tools for analyzing genome data, and disseminates biomedical information - all for the better understanding of molecular processes affecting human health and disease.

The Entrez system can provide views of gene and protein sequences and chromosome maps. Entrez can efficiently retrieve related sequences, structures, and references. Some textbooks are also available online through the Entrez system. Entrez Global Query is an integrated search and retrieval system that provides access to all databases simultaneously.

The sequences of the surface markers VEGFR 1, 2 and 3 were retrieved in FASTA format.

VEGFR 1

Source: Homo sapiens (Human)

Primary accession number

P17948

Source: Mus musculus (Mouse)

Primary accession number

P35969

Source: Rattus norvegicus (Rat)

Primary accession number

P53767 
VEGFR 2

Source: Homo sapiens (Human)

Primary accession number

P35968

Source: Mus musculus (Mouse).

Primary accession number

P35918

Source: Rattus norvegicus (Rat).

Primary accession number

O08775

V EGF 3

Source: Homo sapiens (Human)

Primary accession number

P35916

Source: Mus musculus (Mouse).

Primary accession number

P35917

Source: Rattus norvegicus (Rat)

Primary accession number

Q91ZT1

Multiple sequence Alignment:-

The retrieved sequences were aligned using DNAMAN Software.

DNAMAN Software. DNAMAN carries out multiple sequence alignment, designing PCR primers, protein sequence analysis or drawing plasmids. DNAMAN's speed, flexibility, accuracy and high quality presentations make it one of the fundamental tools used extensively in molecular biology. We used the Multiple Alignment Sequence Editor of DNAMAN (MASED) as an efficient tool for multiple sequence editing. The result of a multiple alignment is loaded directly into MASED. MASED can recognize DNAMAN multiple sequence format (MSD format), GCG/MSF format, Clustal format and GDE format. DNAMAN can open MSD file directly into MASED, other format files must be opened with the File | Open Special | Multiple Alignment command. The multiple alignment editor can output an alignment in different formats: GCG/MSF, CLUSTAL, NBRF/PIR, and GDE. The multiple input and out put capacity of DNAMAN makes it compatible with major sequence analysis software.

\section{Producing Trees:-}

MASED can produce multiple alignment trees. It calculates the homology matrix and establishes related distances between all pairs of sequences. Bootstrapping of a phylogenetic tree.Bootstrap test can be carried out for the confidence values on the phylogenetic tree.DNAMAN calculates the homology matrix and establishes related distances between all pairs of sequences. Consequently, DNAMAN can output a distance matrix of multiple alignment, and draw phylogenetic trees or homology trees. Bootstrapping tests can be carried out for the confidence value of a phylogenetic tree.

\section{Secondary structure prediction for proteins:-}

Secondary Structure Prediction was performed for the human VEGFR sequences using HNN - Hierarchical Neural Network method (Guermeur, 1997).Expression provides an interface to a large range of sophisticated secondary structure prediction algorithms. Protein Expression presently supports nine different secondary structure prediction algorithms. All computations are performed via the Network Protein Sequence Analysis server (PBIL, France). In this study, the prediction method followed was The HNN (Hierarchical Neural Network) prediction method.

\section{Results and Discussion:-}

Multiple Sequence Alignment of the retrieved sequences was obtained using DNAMAN Software. The VEGFR sequences within each class were compared for homology between the sequences from different origins.

Vascular endothelial growth factor receptor 1:-

The VEGFR 1 sequences from Homo sapiens (Human), Mus musculus (Mouse), Rattus norvegicus (Rat) were aligned as follows.

FILE 1: Multiple_Sequence_Alignment

PROJECT:

NUMBER: 3

MAXLENGTH: 1710

NAMES: P17948-VEGFR1-human P35969-VEGFR1-mouse P53767-VEGFR1-rat

MAXNAMELEN: 18 
ORIGIN (Only representative portions)

P17948-VEGFR1-human DCTYPEHTMLPUBLICWCDTDHTMLTRANSITINALENSA 40

P35969-VEGFR1-mouse DCTYPEHTMLPUBLICWCDTDHTMLTRANSITINALENSA 40

P53767-VEGFR1-rat DCTYPEHTMLPUBLICWCDTDHTMLTRANSITINALENSA 40

Consensus detypehtmlpublicwcdtdhtmltransitinalensa

P17948-VEGFR1-human .MVSYWDTGVLLCALLSCLLLTGSSSGSKLKDPELSLKGT 381

P35969-VEGFR1-mouse eMVScWDTaVLpyALLgCLLLTGygSGSKLKvPELSLKGT 340

P53767-VEGFR1-rat .MVScWDTaVLpCALLgCLLLTGycSGSKLKgPELSLKGT 321

Consensus mvs wdt vl all cllltg sgsklk pelslkgt

Table 1:- For VEGFR1 sequences, the Identity Percentage between Homo sapiens (Human), Mus musculus (Mouse) nd Rattus norvegicus (Rat) was found to be $\mathbf{8 7 . 5 8 \%}$.

\begin{tabular}{|l|l|l|}
\hline SEQUENCE COMPARED & ORIGIN & $\begin{array}{l}\text { HOMOLOGY } \\
\text { PERCENTAGE }\end{array}$ \\
\hline $\begin{array}{l}\text { Vascular Endothelial Growth Factor } \\
\text { Receptor 1 }\end{array}$ & Homo sapiens (Human) & \\
\cline { 2 - 2 } & Mus musculus (Mouse) & \\
\cline { 2 - 2 } & Rattus norvegicus (Rat) & \\
\hline
\end{tabular}

Table 2:- On comparison of the individual VEGFR1 sequences with each other it was found that the homology between sequences from rat and mouse was greater (91.57\%), when compared to the homology between human and mouse sequences $(\mathbf{8 0 . 9 2 \%})$ and the homology between human and rat sequences $\mathbf{( 8 1 . 1 2 \% )}$ ).

\begin{tabular}{|c|c|c|}
\hline SEQUENCE COMPARED & ORIGIN & $\begin{array}{l}\text { HOMOLOGY } \\
\text { PERCENTAGE }\end{array}$ \\
\hline \multirow[t]{3}{*}{$\begin{array}{l}\text { Vascular Endothelial Growth Factor } \\
\text { Receptor 1 }\end{array}$} & $\begin{array}{l}\text { Homo sapiens (Human) and Mus } \\
\text { musculus (Mouse) }\end{array}$ & $80.92 \%$ \\
\hline & $\begin{array}{l}\text { Mus musculus (Mouse) and Rattus } \\
\text { norvegicus (Rat) }\end{array}$ & $91.57 \%$ \\
\hline & $\begin{array}{l}\begin{array}{l}\text { Rattus norvegicus (Rat) and Homo } \\
\text { sapiens (Human) }\end{array} \\
\text { (Hom }\end{array}$ & $81.12 \%$ \\
\hline
\end{tabular}

Vascular endothelial growth factor receptor 2:-

The VEGFR 2 sequences from Homo sapiens (Human), Mus musculus (Mouse), Rattus norvegicus (Rat) were aligned as follows.

FILE 2: Multiple_Sequence_Alignment

PROJECT:

NUMBER: 3

MAXLENGTH: 1708

NAMES: P35968-VEGFR2-human P35918-VEGFR2-mouse O08775-VEGFR2-rat

MAXNAMELEN: 18

ORIGIN (Only representative portions)

P35968-VEGFR2-human DCTYPEHTMLPUBLICWCDTDHTMLTRANSITINALENSA 40

P35918-VEGFR2-mouse DCTYPEHTMLPUBLICWCDTDHTMLTRANSITINALENSA 40

O08775-VEGFR2-rat DCTYPEHTMLPUBLICWCDTDHTMLTRANSITINALENSA 40

Consensus dctypehtmlpublicwcdtdhtmltransitinalensa

P35968-VEGFR2-human VEVTECSDGL..FCKTLTIPKVIGNDTGAYKCFYRETDLA 421

P35918-VEGFR2-mouse VIVTECggGdsiFCKTLTIPrVvGNDTGAYKCsYRdvDiA 415

O08775-VEGFR2-rat VlVTECg..dsiFCKTLTvPrVvGNDTGAYKCFYRdTDvs 404

Consensus $\quad \mathrm{v}$ vtec fcktlt $\mathrm{p} v$ gndtgaykc yr $\mathrm{d}$ 
Table 3:- For VEGFR 2 sequences, the Identity Percentage between Homo sapiens (Human), Mus musculus (Mouse) and Rattus norvegicus (Rat) was found to be $\mathbf{9 0 . 1 4 \%}$

\begin{tabular}{|l|l|l|}
\hline SEQUENCE COMPARED & ORIGIN & $\begin{array}{l}\text { HOMOLOGY } \\
\text { PERCENTAGE }\end{array}$ \\
\hline Vascular Endothelial Growth Factor Receptor 2 & Homo sapiens (Human) & $\mathbf{9 0 . 1 4 \%}$ \\
\cline { 2 - 3 } & Mus musculus (Mouse) & \\
\cline { 2 - 3 } & Rattus norvegicus (Rat) & \\
\hline
\end{tabular}

Table 4:- On comparison of the individual VEGFR 2 sequences with each other it was found that the homology between sequences from rat and mouse was greater $\mathbf{( 9 2 . 4 4 \% ) , ~ w h e n ~ c o m p a r e d ~ t o ~ t h e ~ h o m o l o g y ~ b e t w e e n ~ h u m a n ~ a n d ~}$ mouse sequences $(\mathbf{8 3 . 3 6 \%})$ and the homology between human and rat sequences $(\mathbf{8 2 . 8 5 \%})$

\begin{tabular}{|c|c|c|}
\hline SEQUENCE COMPARED & ORIGIN & $\begin{array}{l}\text { HOMOLOGY } \\
\text { PERCENTAGE }\end{array}$ \\
\hline \multirow[t]{3}{*}{$\begin{array}{l}\text { Vascular Endothelial Growth Factor } \\
\text { Receptor } 2\end{array}$} & $\begin{array}{l}\text { Homo sapiens (Human) and Mus } \\
\text { musculus (Mouse) }\end{array}$ & $83.36 \%$ \\
\hline & $\begin{array}{l}\text { Mus musculus (Mouse) and Rattus } \\
\text { norvegicus (Rat) }\end{array}$ & $92.44 \%$ \\
\hline & $\begin{array}{l}\text { Rattus norvegicus (Rat) and Homo } \\
\text { sapiens (Human) }\end{array}$ & $82.85 \%$ \\
\hline
\end{tabular}

vascular endothelial growth factor receptor 3:-

The VEGFR 3 sequences from Homo sapiens (Human), Mus musculus (Mouse), Rattus norvegicus (Rat) were aligned as follows.

FILE 3: Multiple_Sequence_Alignment

PROJECT:

NUMBER: 3

MAXLENGTH: 1657

NAMES: P35916-VEGFR3-human P35917-VEGFR3-mouse Q91ZT1-VEGFR3-rat

MAXNAMELEN: 18

ORIGIN (Only representative portions)

P35916-VEGFR3-human DCTYPEHTMLPUBLICWCDTDHTMLTRANSITINALENSA 40 P35917-VEGFR3-mouse DCTYPEHTMLPUBLICWCDTDHTMLTRANSITINALENSA 40 Q91ZT1-VEGFR3-rat DCTYPEHTMLPUBLICWCDTDHTMLTRANSITINALENSA 40

Consensus dctypehtmlpublicwcdtdhtmltransitinalensa

P35916-VEGFR3-human RGRFRAMVELARLDRRRPGSSDRVLFARFSKTEGGARRAS 1273

P35917-VEGFR3-mouse RrRFRAMVEgAkaDRRRPGSSDRaLFtRFlmgkGsARRAp 1273

Q91ZT1-VEGFR3-rat RrRFRAMVEgAkaDRRRIGStDRaLFtRFlmgkGsARRAp 1279

Consensus $\quad \mathrm{r}$ rframve a drrr gs dr lf rf $\mathrm{g}$ arra

Table 5:- For VEGFR 3 sequences, the Identity Percentage between Homo sapiens (Human), Mus musculus (Mouse) and Rattus norvegicus (Rat) was found to be $\mathbf{9 3 . 2 4 \% . ~}$

\begin{tabular}{|l|l|l|}
\hline SEQUENCE COMPARED & ORIGIN & $\begin{array}{l}\text { HOMOLOGY } \\
\text { PERCENTAGE }\end{array}$ \\
\hline Vascular Endothelial Growth Factor Receptor 3 & Homo sapiens (Human) & 93.24\% \\
\cline { 2 - 2 } & Mus musculus (Mouse) & \\
\cline { 2 - 3 } & Rattus norvegicus (Rat) & \\
\hline
\end{tabular}


Table 6:- On comparison of the individual VEGFR3 sequences with each other it was found that the homology between sequences from rat and mouse was greater $\mathbf{( 9 5 . 7 8 \%}$ ), when compared to the homology between human and mouse sequences $(\mathbf{8 5 . 4 6 \%})$ and the homology between human and rat sequences $(\mathbf{8 4 . 6 0 \%})$.

\begin{tabular}{|ll|l|l|l|}
\hline SEQUENCE COMPARED & ORIGIN & $\begin{array}{l}\text { HOMOLOGY } \\
\text { PERCENTAGE }\end{array}$ \\
\hline $\begin{array}{l}\text { Vascular Endothelial Growth Factor } \\
\text { Receptor 3 }\end{array}$ & $\begin{array}{l}\text { Homo sapiens (Human) and Mus } \\
\text { musculus (Mouse) }\end{array}$ & $\begin{array}{l}\text { 85.46\% } \\
\text { Mus musculus (Mouse) and Rattus } \\
\text { norvegicus (Rat) }\end{array}$ & $\mathbf{9 5 . 7 8 \%}$ \\
\cline { 2 - 2 } & $\mathbf{8 4 . 6 0 \%}$ \\
sapiens (Human)
\end{tabular}

\section{Phylogenetic Analysis:-}

The phylogeny between the VEGFR sequences retrieved from various sources was established by plotting trees and the results were obtained as follows.

Vascular endothelial growth factor receptor 1:-

P17948-VEGFR1-human: 0.12358

P35969-VEGFR1-mouse: 0.03837

P53767-VEGFR1-rat: 0.03183

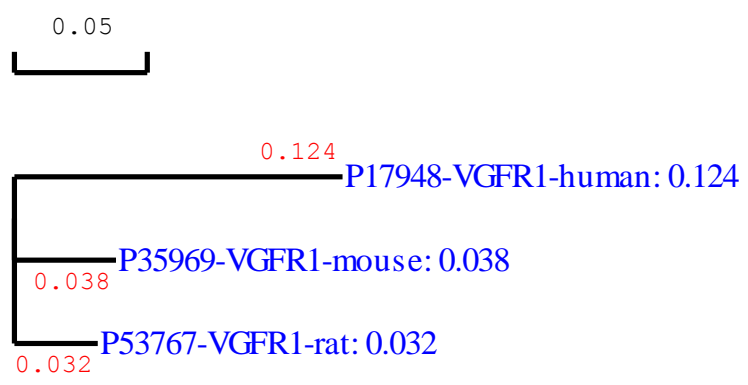

Figure 1:- Phylogenetic tree of VEGFR 1

Vascular endothelial growth factor receptor 2;-

P35968-VEGFR2-human: 0.10447

P35918-VEGFR2-mouse: 0.02910

O08775-VEGFR2-rat: 0.02315
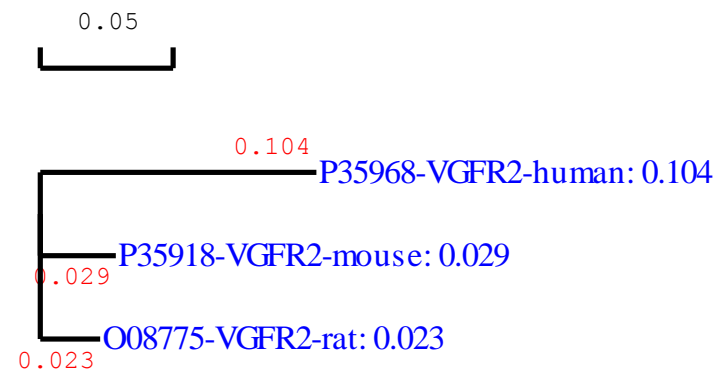

Figure 2:- Phylogenetic tree of VEGFR 2

\section{VASCULAR ENDOTHELIAL GROWTH FACTOR RECEPTOR 3}

P35916-VEGFR3-human: 0.09399

P35917-VEGFR3-mouse: 0.01550

Q91ZT1-VEGFR3-rat: 0.02210 


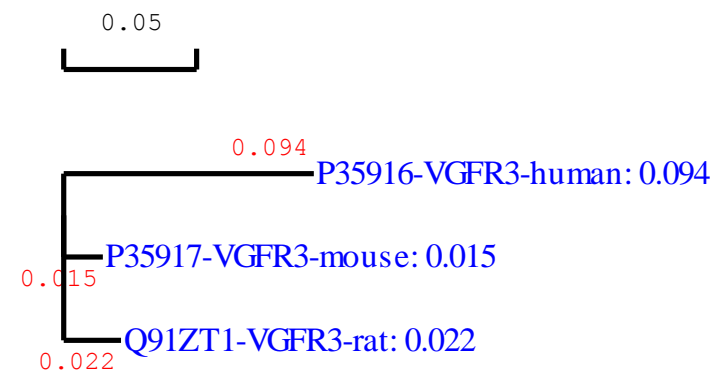

Figure 3:- Phylogenetic tree of VEGFR 3

The phylogenetic tree for VEGFR1 depicts that the sequences from Mus musculus (Mouse) and Rattus norvegicus (Rat) display greater similarity than with each of the sequences in comparison with Homo sapiens (Human) VEGFR 1 sequence. Similar results were obtained in case of phylogenetic tree generated between VEGFR2 as well as VEGFR 3 sequences of Homo sapiens (Human), Mus musculus (Mouse) and Rattus norvegicus (Rat).

\section{Secondary structure prediction for human vegfr:-}

Secondary structure prediction was done for the Human VEGFR sequences using HNN Secondary Structure Prediction Method and the results were obtained as follows.

Date Set 1:- Human vegfr1: Hierarchical Neural Network result for: UNK_129930
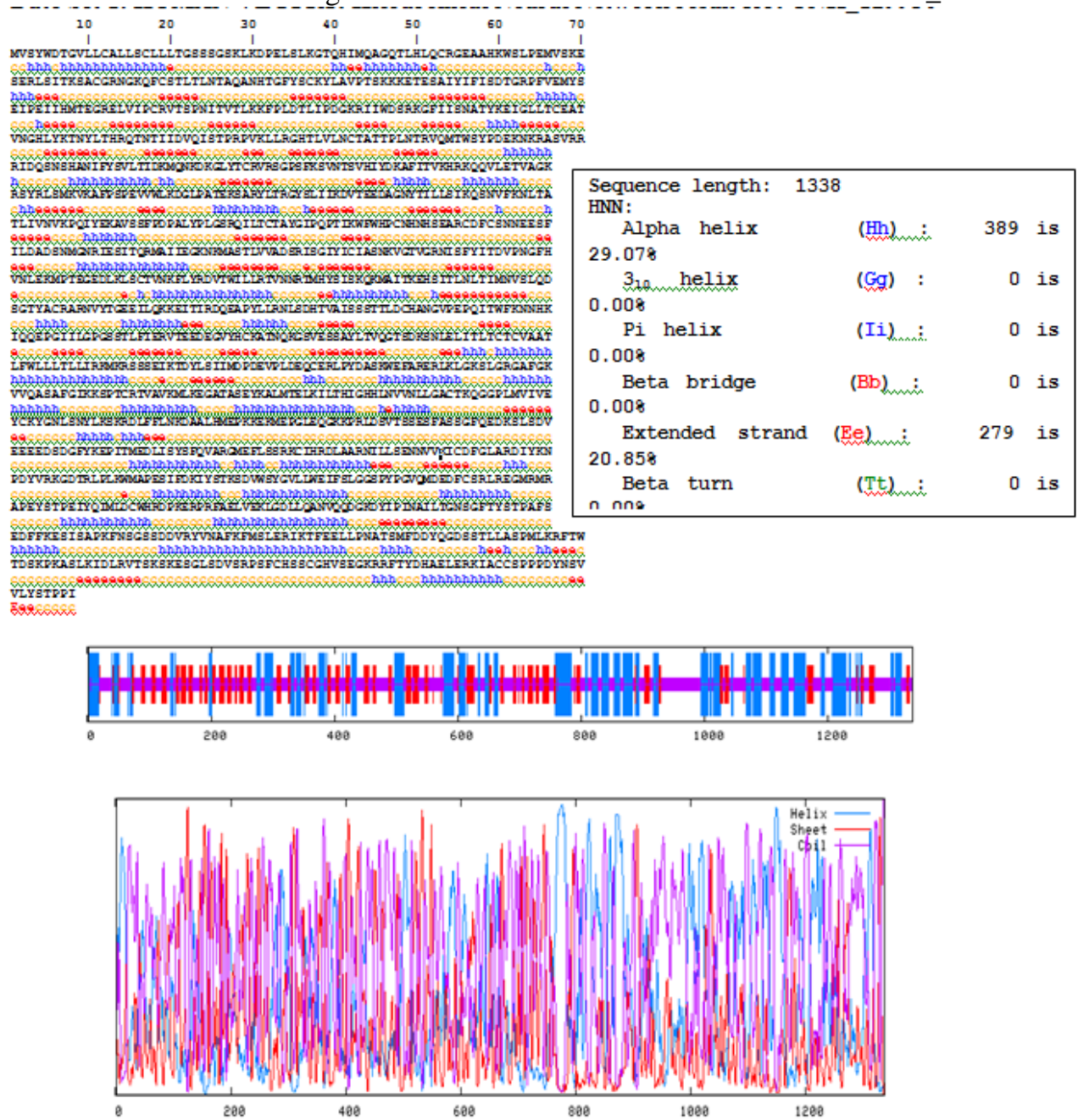

Date Set 1. Using HNN for VEGFR 1 sequence, the length of the sequence was found to be 1338 . The protein is chiefly folded into random coils $(50.07 \%)$ and also consists of alpha helix $(29.07 \%)$ and extended strand (20.85\%). 
Date Set 2:- HUMAN VEGFR2: Hierarchical Neural Network result for : UNK_145240

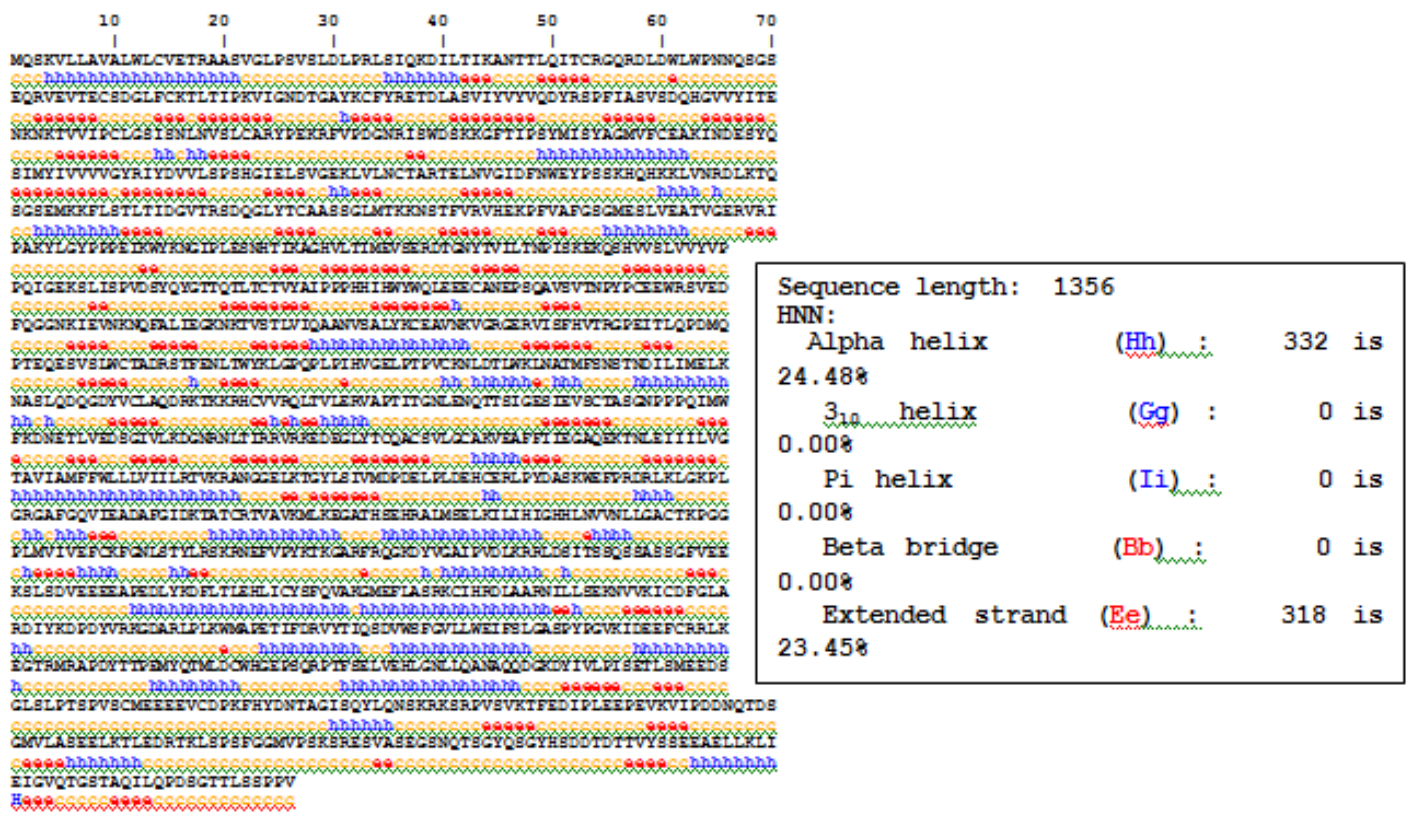
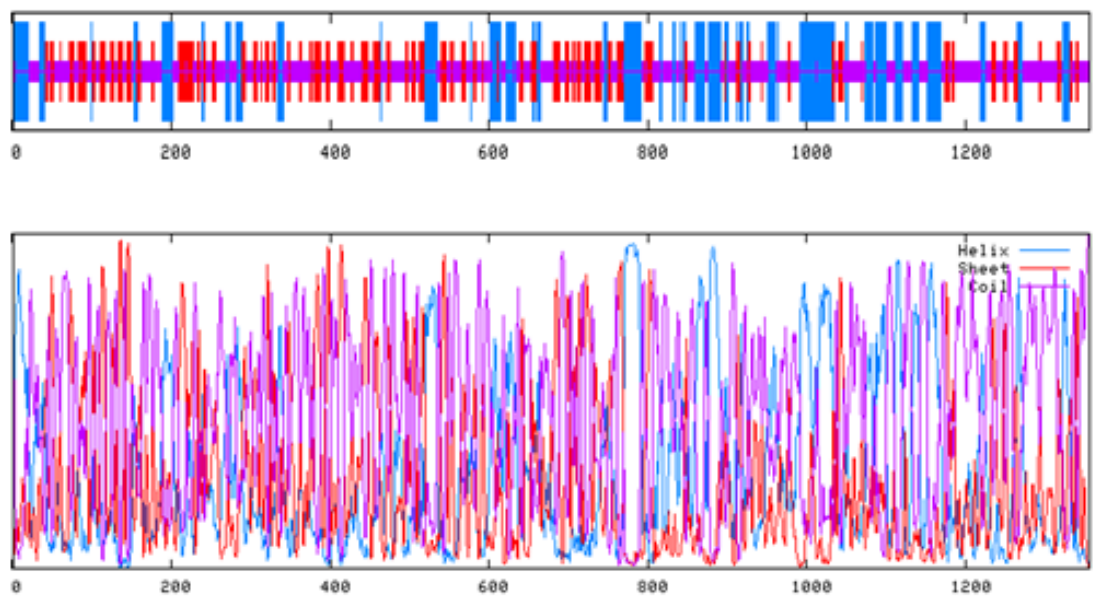

Data Set 2.Using HNN for VEGFR 2 sequence, the length of the sequence was found to be 1356. The protein is chiefly folded into random coils (52.06\%) and also consists of alpha helix (24.48\%) and extended strand (23.45\%). 
Data Set 3. HUMAN VEGFR3: Hierarchical Neural Network result for: UNK_154230

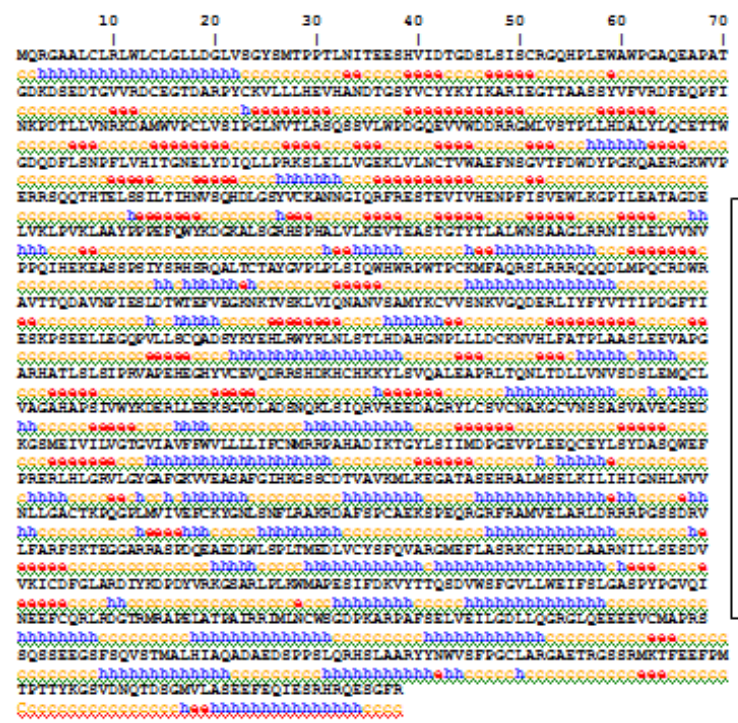

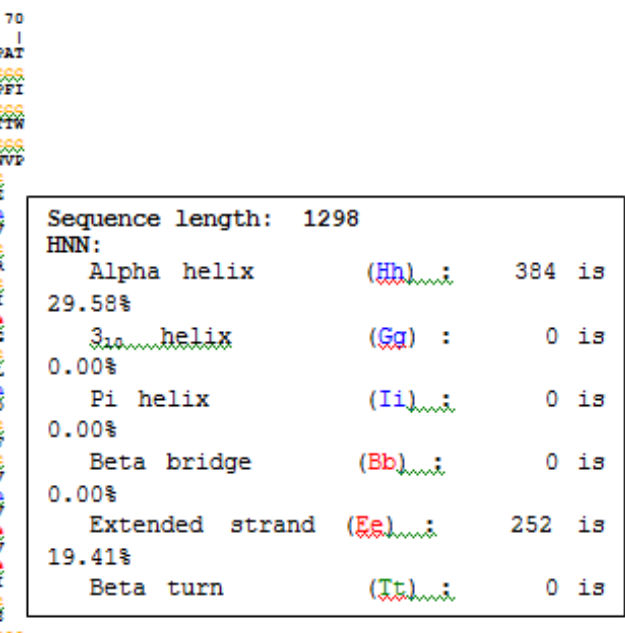


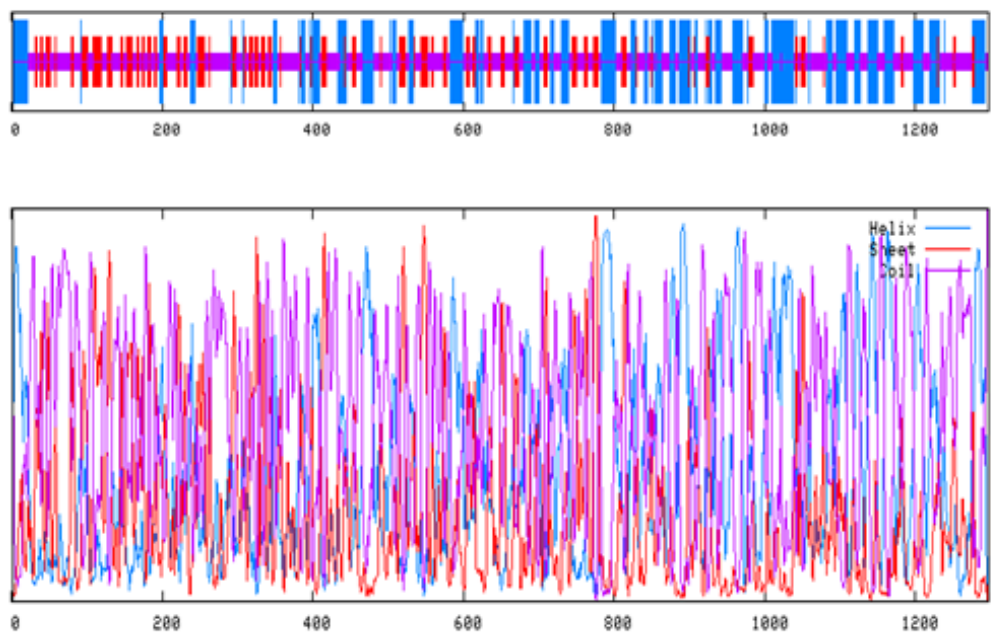

Data Set 3. Using HNN for VEGFR sequence, the length of the sequence was found to be 1298. The protein is chiefly folded into random coils (51.00\%) and also consists of alpha helix (29.58\%) and extended strand (19.41\%).

\section{Discussion:-}

Multiple sequence Alignment predicted the degree of variation of VEGFR 1 at genus levels:-

Multiple sequence alignment (MSA) is an indispensable tool for studying the difference and homology of the macromolecules. There are many studies which compare with the protein sequence among different genus and species (Ramu Chenna et al., 2003). Therefore MSA becomes useful to know the difference in the amino acid composition which further gives the details of protein confirmation necessary for the functions like binding, activation etc. The current information of the VEGFR sequences retrieved from each species were assessed for homology using DNAMAN Software. The secondary protein structure further predicted showed the variations among each of the VEFG1, 2, and 3 subtypes. More over the results are mostly from consistency-based programs with higher accuracy determined the reliability of the results. TheVEGFR1 sequences showed an identity percentage of 87.58\% between humans, mouse and rat (Table 1). On comparison of the individual VEGFR1 sequences among the species, we observed the homology between sequences from rat and mouse was $91.57 \%$ compared to the $80.92 \%$ homology between human and mouse and $81.12 \%$ homology between human and rat sequences respectively (Table 2). 
In the case of VEGFR2 sequences, the indent percentage was found to be $90.14 \%$. (Table 3)among human, mouse and rat. The individual comparison VEGFR2sequences homology between rat and mouse was $92.44 \%$, compared to the $83.36 \%$ homology between human and mouse and $82.85 \%$ homology between human and rat sequences (Table 4). The overall homology percentage for VEGFR 3 among the human, mouse and rat was $93.24 \%$ (Table 5). The sequence homology between rat and mouse is $95.78 \%$ compared to $85.46 \%$ human and mouse and $84.60 \%$ human and rat (Table 6). The homology between the VEGFR 1, 2 and 3 sequences from mouse and rat were more homogenous compared to humans showing the higher percent of variation with highly evolved vertebrate (Higgins DG et al 1996).

\section{Phylogenetic analysis of VEGFR 1,2 \&3 reveals the evolutionary relationship between humans and murine species:- \\ Phylogenetic trees were drawn using DNAMAN Software to establish the homology of the sequences from different sources. The algorithm and results were in compliance with the previous published works (Xin-Rong Liu et al., 2004) on evolutionary relationships and similarities among proteins.The phylogenetic tree for VEGFR1 depicts that the sequences from mouse and rat display greater similarity than with each of the sequences in comparison with humans (Figure1) Similar results were obtained in case of phylogenetic tree generated between VEGFR2 (Figure 2) as well as VEGFR 3 (Figure 3) sequences of human, mouse and rat (Hernández-García et al.,2015).}

Secondary structure of VEFG prediction shows the variation on the protein confirmation across species:Secondary Structure prediction for human VEGFR sequences using Hierarchical Neural Network Secondary Structure Prediction Method was on par with the previous studies (Guermeur Y, 1997, Chandrasekaran V et al., 2007) and the their proposed this algorithm for secondary structure for proteins.Using HNN for VEGFR 1 sequence, the length of the sequence was found to be 1338 . The $50.07 \%$ protein is chiefly folded into random coils with 29.07\% of alpha helix and $20.85 \%$ as extended strand (Data set 1).The length of the VEGFR 2 sequence was 1356. $52.06 \%$ protein is chiefly folded into random coils with $24.48 \%$ of alpha helix and $23,45 \%$ of extended strand (Data set 2). The length if the VEGFR 3 sequence was 1928. 51.00\% protein is chiefly folded into random coils with $29.58 \%$ as alpha helix $(29.58 \%)$ and $19.41 \%$ extended strand (Data set3). Thus, secondary structure prediction reveals the structural similarity between the classes of VEGFR sequences. All classes of VEGFRs reveal that large regions of their secondary structure consist of random coils and the remainder is folded into alpha helix (Wu G et al.,2013).

\section{Conclusion:-}

The MSCs VEGFR 1, 2 and 3 show homology and identity upon multiple sequence alignment. The sequences retrieved from Mus musculus (Mouse) and Rattus norvegicus (Rat) show greater homology in comparison with Homo sapiens (Human). The Phylogenetic Tree asserts the similarity and homology among VEGFR sequences from different species. Secondary structure prediction of the VEGFR sequences from Homo sapiens (Human) revealed that all the three receptors, i.e., VEGFR1, VEGFR2 and VEGFR3 show about 50\% similarities in their secondary structure in random coils while remaining regions exist either as alpha helices or as extended strands.

\section{Reference:-}

1. Amos Bairoch, Rolf Apweiler. The SWISS-PROT protein sequence database and its supplement TrEMBL in 2000. Nucleic Acids Research, 2000; 28:1: 45-48.

2. Adel Alhadlaq, Jeremy J. Mao. Mesenchymal Stem Cells: Isolation and Therapeutics. Endocrinology. 2004; 145(11):4838-4845.

3. Chandrasekaran V, Ambati J, Ambati BK, Taylor EW. Molecular docking andanalysis of interactions between vascular endothelial growth factor (VEGF) andSPARC protein. J Mol Graph Model. 2007 Nov;26(4):775-82.

4. Choudhery MS, Badowski M, Muise A, Pierce J, Harris DT. Subcutaneous AdiposeTissue-Derived Stem Cell Utility Is Independent of Anatomical Harvest Site.Biores Open Access. 2015 Feb 1;4(1):131-45.

5. Guermeur Y. Combinaison de classifieurs statistiques, Application a la prediction de structure secondaire des proteines. PhD Thesis, 1997.

6. Higgins DG, Thompson JD, Gibson TJ. Using CLUSTAL for multiple sequence alignments. Methods Enzymol. 1996;266:383-402.

7. Hernández-García R, Iruela-Arispe ML, Reyes-Cruz G, Vázquez-Prado J.Endothelial RhoGEFs: A systematic analysis of their expression profiles inVEGF-stimulated and tumor endothelial cells. Vascul Pharmacol. 2015 Nov;74:60-72. 
8. Igarashi Y, Chosa N, Sawada S, Kondo H, Yaegashi T, Ishisaki A. VEGF-C andTGF- $\beta$ reciprocally regulate mesenchymal stem cell commitment to differentiationinto lymphatic endothelial or osteoblastic phenotypes. Int J Mol Med. 2016Apr;37(4):1005-13.

9. Kasper G, Dankert N, Tuischer J, Hoeft M, Gaber T, Glaeser JD, Zander D,Tschirschmann M, Thompson M, Matziolis G, Duda GN. Mesenchymal stem cellsregulate angiogenesis according to their mechanical environment. Stem Cells. 2007 Apr;25(4):903-10.

10. Kobolak J, Dinnyes A, Memic A, Khademhosseini A, Mobasheri A. Mesenchymal stem cells: Identification, phenotypic characterization, biological properties andpotential for regenerative medicine through biomaterial micro-engineering oftheir niche. Methods. 2016 Apr 15;99:62-8.

11. Louveau I, Perruchot MH, Bonnet M, Gondret F. Invited review: Pre- andpostnatal adipose tissue development in farm animals: from stem cells toadipocyte physiology. Animal. 2016; 10(11):1839-1847.

12. Matthias Clauss, Herbert Weich, Georg Breier, Ulrike Knies, Wolfgang Röckl, Johannes Waltenberger and Werner Risau. The Vascular Endothelial Growth Factor Receptor Flt-1 Mediates Biological Activities Implications for a functional role of placenta growth factor in monocyte activation and chemotaxis. J. Biol.Chem Volume. 1996; 271:30;17629-17634

13. Ramu Chenna, Hideaki Sugawara, Tadashi Koike, Rodrigo Lopez, Toby J. Gibson, Desmond G. Higgins and Julie D. Thompson Multiple sequence alignment with the Clustal series of programs. Nucleic Acids Research. 2003; 31:13:3497-3500.

14. Sayers EW, Karsch-Mizrachi I. Using GenBank. Methods Mol Biol. 2016;1374:1-22.

15. Totsugawa T, Kobayashi N, Okitsu T, Noguchi H, Watanabe T, Matsumura T, Maruyama M, Fujiwara T, Sakaguchi M, Tanaka N. Lentiviral transfer of the LacZgene into human endothelial cells and human bone marrow mesenchymal stem cells.Cell Transplant. 2002;11(5):481-8.

16. Wu G, Han K, Lv F. Use of fast conformational sampling to improve thecharacterization of VEGF A-peptide interactions. J Theor Biol. 2013 Jan21;317:293-300.

17. Xin-Rong Liu, Rou-Li Zhou, Qing-Yun Zhang, Ye Zhang, Yue-Ying Jin, Ming Lin, Jing-An Rui, Da-Xiong Ye. Structure analysis and expressions of a novel tetratransmembrane protein, lysosome-associated protein transmembrane 4 B associated with hepatocellular carcinoma. World J Gastroenterol 2004;10(11):1555-1559 